\section{September 2004}

JPGM GOLD CON: 50 Years of Medical Writing

International conference on journal writing and publishing: September 23-26, 2004, Mumbai, India. Address for information: Dr Atul Goel, Department of Neurosurgery, Seth G. S. Medical College, Parel, Mumbai-400 012, India (telephone: 91-2224129884; fax: 91-22-25032398; E-mail: goldcon@jpgmonline.com).

\section{October 2004}

Controversies in Adult Cardiac

Surgery: The Fourth in a Series Symposium: October 7-8, 2004, Santa Monica, California, the Loews Santa Monica Beach Hotel. Address for information: Promedica International, a California Corporation, 7777 Center Ave, Suite 500, Huntington Beach, CA 92647 (telephone: 714-799-1617, ext 3; fax: 714-799-1686; E-mail: education@promedica-intl.com; Web site: http://www.promedica-intl.com).

\section{Heart Failure \& Rotary Blood Pump Summit}

Summit: October 7-10, 2004, Cleveland, Ohio, InterContinental Hotel and MBNA Conference Center, presented by The Cleveland Clinic, George M. \& Linda H. Kaufman Center for Heart Failure, in cooperation with The International Society for Rotary Blood Pumps. For information: The Cleveland Clinic Center for Continuing Education (telephone: 216-4445696; 800-762-8173; Web site: www. clevelandclinicmeded.com/heartfailurepump. htm).

significant activities must be ceived at least 8 weeks before the desired month of publication. Information will be limited to title of meeting, date, place, and an address to obtain further information. Send announcements to Pamela W. Fried, Editorial Office, The Journal of Thoracic and Cardiovascular Surgery, MCP Hahnemann University, 245 N 15th St, Mail Stop 496, Philadelphia, PA 19102-1192.

\section{Congenital Cardiovascular Surgery} Symposium: San Diego 2004

Symposium: October 15-16, 2004, San Diego, California, Manchester Grand Hyatt Regency Hotel. Address for information: The Continuing Medical Education, Children's Hospital and Health Center (telephone: 888-892-9249; Web site: www. chsd.org/cme). 57th Annual Meeting of the Japanese Association for Thoracic Surgery Meeting: October 20-22, 2004, Sapporo, Japan, Royton Sapporo Hotel: Address for information: Professor Tomio Abe, MD, Sapporo Medical University, South 1, West 16, Chuo-ku, Sapporo, Japan 060-8543 (Japan Convention Services, Inc: telephone: +81-11-738-3503; fax: +81-11-738-3504; E-mail: 57thjats@convention.co.jp; Web site: www2.convention.co.jp/57thjats/).

\section{December 2004}

Fifth International Symposium on Pediatric Cardiac Intensive Care Symposium: December 1-4, 2004, Miami, Florida, Four Seasons Hotel. Presented by the Texas Children's Hospital Heart Center and the Pediatric Cardiac Intensive Care Society (PCICS). For information: E-mail: info@PCICsymposium.org; Web site: www.PCICsymposium.org.

4th Meeting of Mechanical Circulatory Support-Today's Facts and Future Trends

Meeting: December 1-4, 2004, Bad Oeynhausen, Germany. Address for information: Susanne Traut, Heart Center NRW, Georgstr 11, 32545 Bad Oeynhausen, Germany (telephone: +49-5731-971333; fax: +495731-971820; E-mail: straut@hdz-nrw.de).

Cardiac \& General Thoracic Surgery Update 2004

Meeting: December 10-12, 2004, Scottsdale, Arizona, Westin Kierland Resort \& Spa. For information: Member Services. Telephone: 800-343-2227 (in the United States); 847-498-1400 (outside the United States); Web site: www.chestnet.org.
Al Bassel Heart Society 2nd Meeting Meeting: December 4-7, 2004, Damascus, Syria, Dummar Housing Project. Address for information: Al Bassel Heart Institute, Damascus, Syria, Dummar Housing Project (telephone: 00963113121471 ; fax:00963 11 3121477; E-mail: bhi-syr@ net.sy). 


\section{February 2005}

13th Annual Meeting of the Asian Society for Cardiovascular Surgery Congress: February 5-8, 2005, Chiang Mai, Thailand. Address for information: Supreecha Tanamai, MD, Honorary Secretary, Institute of Cardiovascular Diseases, Rajavithi Hospital, 2 Rajavithi Rd, Bangkok 10400, Thailand (telephone: 662354-8006; fax: 662-354-8007; E-mail: office@ascvs2005.org; Web site: http:// www.ascvs2005.org).

\section{March 2005}

\section{Cardiothoracic Techniques \& \\ Technologies (CTT) 11th Annual Meeting}

Meeting: March 3-5, 2005, Contemporary Resorts, Walt Disney Parks, Florida. For information: CTT Teri Valls (telephone: 305-663-1628; fax: 305-663-1644; E-mail: tvalls@meccinc.com; Web site: cttweb. net).

\section{CTT 2005, Cardiothoracic Techniques} and Technologies

11th Annual Meeting, March 4-6, 2005, Lake Buena Vista, Florida, The Contemporary Resort, Walt Disney World Parks and Resorts, sponsored by the Foundation for Advancement of Cardiac Techniques and Technologies, a nonprofit organization. $A d$ dress for information: Teri Valls, CMP, CMM, CTT Administrative Office, 1550 South Dixie Highway, Suite 214, Coral Gables, FL 33146 (telephone: 305-6631628; fax: 305-663-1644; E-mail: tvalls@ meccinc.com; Web site: www.cttweb.net).

The Society of Cardiothoracic Surgeons of Great Britain and Ireland

Annual General Meeting: March 5-8, 2005, Kensington, London, United Kingdom, The Olympia Conference Centre. Abstract submission online at www.scts.org until midnight, November 5, 2004. For information: sctsadmin@scts.org.

20th Annual Interventional Cardiology 2005: The International Symposium Symposium: March 21-25, 2005, Snowmass Village, Colorado, the Silvertree Hotel. Address for information: Promedica International, a California Corporation, 7777
Center Ave, Suite 500, Huntington Beach, CA 92647 (telephone: 714-799-1617, ext 3; fax: 714-799-1686; E-mail: education@promedica-intl.com; Web site: http://www.promedica-intl.com).

\section{April 2005}

The American Association for Thoracic Surgery

Annual Meeting, April 10-13, 2005, San Francisco, California, Moscone Convention Center. Address for information: 900 Cummings Center, Suite 221-U, Beverly, MA 01915 (fax: 978-524-8890; E-mail: aats@prri.com. Web site: www.aats.org).

\section{May 2005}

The First International Conference on Pediatric Mechanical Circulatory Support Systems, Pediatric Heart Transplantation, and Pediatric Cardiopulmonary Perfusion Conference: May 19-21, 2005, Hershey, Pennsylvania, the Hotel Hershey (sponsored by Penn State College of Medicine). For information: www.hmc.psu.edu/ce/ pediatrics.htm.

\section{4th International Congress of the} European Society for CardioVascular Surgery

Congress: May 19-22, 2005, Athens, Greece (http://www.escvsathens2005.org). Addresses for information: Scientific information: Dr George M. Palatianos, MD, $\mathrm{PhD}$, Onassis Cardiac Surgery Center, 356 Sygrou Ave, 17674 Athens, Greece (telephone: +30-210-9493314; fax: +30-2109493331; E-mail: palatianos@otenet.gr); Conference Secretariat: Ms Liana Iliopoulou, Triaena Tours and Congresses SA, Atchley House, 15, Mesogion Ave, 11526 Athens, Greece (telephone +30-2107499317; fax: +30-210-7705752; E-mail: lianae@triaenatours.gr).

\section{June 2005}

\section{Society for Heart Valve Disease}

Third Biennial Meeting: June 17-20, 2005, Vancouver, British Columbia, Canada (abstract deadline: January 31, 2005). For information: Executive Secretariat: telephone: 44207351 8534; fax: 44207351
8531; E-mail: secretariat@ shvd.org; Web site: www.shvd.org.

\section{The Western Thoracic Surgical}

Association

Annual Meeting: June 22-25, 2005, Victoria, British Columbia, Canada, the Empress Hotel. Address for information: 900 Cummings Center, Suite 221-U, Beverly, MA 01915 (fax: 978-524-8890; E-mail: wtsa@ prri.com).

\section{August 2005}

\section{ACCP: Pulmonary Board Review} Course 2005

Course: August 19-23, 2005, Scottsdale, Arizona, The Westin Kierland Resort \& Spa. Contact name: Member Services (telephone: 1-800-343-2227 US; 1-847498-1400 international; Web site: www. chestnet.org).

\section{ACCP: Critical Care Board Review Course 2005}

Course: August 24-28, 2005, Scottsdale, Arizona, The Westin Kierland Resort \& Spa. Contact name: Member Services (telephone: 1-800-343-2227 US; 1-847498-1400 international; Web site: www. chestnet.org).

\section{September 2005}

Fourth World Congress on Pediatric Cardiology and Cardiac Surgery Congress: September 18-22, 2005, Buenos Aires, Argentina, Sheraton Buenos Aires Hotel \& Convention Center. Address for information: Congress Secretariat c/o Congresos Internacionales S.A., Lima 355 PB "B"-C1073AAG Ciudad de Buenos Aires, Argentina (telephone: +54-114382-5772; fax: +54-11-4382-5730; Web site: www.congresosint.com.ar/pccs2005; E-mail: pccs2005@ congresosint.com.ar).

\section{October 2005}

\section{ACCP: Chest 2005}

Course: October 29-November 2, 2005, Montreal, Quebec, Canada, Montreal Convention Centre. Contact name: Member Services (telephone: 1-800-343-2227 US; 1-847-498-1400 international; Web site: www.chestnet.org). 